\title{
CONDUTAS DOS PROFISSIONAIS DE ENFERMAGEM FRENTE AOS ALARMES DOS VENTILADORES MECÂNICOS EM UMA UNIDADE DE TERAPIA INTENSIVA
}

\section{CONDUCT OF NURSING PROFESSIONALS TO THE ALARMS OF MECHANICAL VENTILATORS IN AN INTENSIVE CARE UNIT}

\section{CONDUCTAS DE LOS PROFESIONALES DE ENFERMERÍA FRENTE A LAS ALARMAS DE LOS VENTILADORES MECÁNICOS EN UNA UNIDAD DE TERAPIA INTENSIVA}

Kamila Azevedo de Souza ${ }^{1}$, Vanessa Galdino de Paula ${ }^{2}$, Adriana Carla Bridi ${ }^{3}$, Flávia Giron Camerini ${ }^{4}$, Andrezza Serpa Franco ${ }^{5}$, Bruna da Silva Louredo Pereira ${ }^{6}$.

\section{RESUMO}

Objetivos: caracterizar quais alarmes sonoros disparados por ventiladores mecânicos foram mais frequentes, descrever o tempo estímulo-resposta aos alarmes do ventilador mecânico e analisar as condutas dos profissionais de enfermagem diante dos alarmes ventilatórios. Método: pesquisa descritiva, quantitativa, realizada em uma unidade de terapia intensiva de um hospital universitário do Rio de Janeiro. Os dados foram tratados com estatística descritiva. Resultados: realizadas 60 horas de observação, nesse período, soaram 25 alarmes de ventiladores mecânicos, 20 alarmes foram atendidos e 5 pararam sem nenhuma intervenção. Os alarmes mais prevalentes foram volume minuto expirado baixo, e pressão de vias aéreas alta. O tempo mínimo para atendimento dos alarmes foi 10 segundos, o tempo máximo 3 minutos, e o tempo médio 38 segundos. As condutas mais realizadas pelos profissionais de enfermagem foram aspiração traqueal, reposicionamento do circuito e ausculta pulmonar. Conclusão: os achados da pesquisa apontaram que, nos alarmes mais prevalentes, as condutas dos profissionais de enfermagem, de acordo com a literatura, não foram suficientes para a resolutividade dos problemas apresentados.

Descritores: Enfermagem; Respiração artificial; Unidades de terapia intensiva; Alarmes clínicos.

\begin{abstract}
Objectives: To characterize which alarms were activated by mechanical ventilators were more frequent, to describe the stimulusresponse time to the mechanical ventilator alarms and to analyze the nursing professionals conducts before ventilatory alarms. Method: descriptive, quantitative research performed in an intensive care unit of a university hospital in Rio de Janeiro. The data were treated with descriptive statistics. Results: 60 hours of observation were performed. During this period, 25 mechanical ventilator alarms sounded, 20 alarms were answered and 5 stopped without any intervention. The most prevalent alarms were low expired minute volume, and high airway pressure. The minimum time to answer the alarms was 10 seconds, the maximum time 3 minutes, and the average time 38 seconds. The most accomplished conducts by the nursing professionals were tracheal aspiration, circuit repositioning and pulmonary auscultation. Conclusion: the research findings pointed out that, in the most prevalent alarms, nursing professionals' conduct, according to the literature, was not enough to solve the problems presented.
\end{abstract}

Descriptors: Nursing; Respiration artificial; Intensive care units; Clinical alarms.

\section{RESUMEN}

Objetivos: caracterizar que alarmas sonoras disparadas por ventiladores mecánicos fueron más frecuentes, describir el tiempo estímulo-respuesta a las alarmas del ventilador mecánico y analizar las conductas de los profesionales de enfermería ante las alarmas ventilatorios. Método: investigación descriptiva, cuantitativa realizada en una unidad de terapia intensiva de un hospital universitario de Río de Janeiro. Los datos fueron tratados con estadística descriptiva. Resultados: realizadas 60 horas de observación, en ese período sonaron 25 alarmas de ventiladores mecánicos, 20 alarmas fueron atendidas y 5 pararon sin ninguna intervención. Las alarmas más prevalentes fueron el volumen minuto expirado bajo, y la presión de las vías aéreas alta. El tiempo mínimo para la atención de las alarmas fue 10 segundos, el tiempo máximo 3 minutos, y el tiempo promedio 38 segundos. Las conductas más realizadas por los profesionales de enfermería fueron aspiración traqueal, reposicionamiento del circuito y auscultación pulmonar. Conclusión: los hallazgos de la investigación apuntaron que, en las alarmas más prevalentes, las conductas de los profesionales de enfermería, de acuerdo con la literatura, no fueron suficientes para la resolución de los problemas presentados.

Descriptores: Enfermería; Respiración artificial; Unidades de cuidados intensivos; Alarmas clínicas.

${ }^{1}$ Graduada em Enfermagem. Residente em Terapia Intensiva pelo Hospital Universitário Pedro Ernesto da UERJ. ${ }^{2}$ Graduada em Enfermagem. Doutoranda em Enfermagem e Biociências pela Universidade Federal do Estado do Rio de Janeiro. Docente na Universidade do Estado do Rio de Janeiro. ${ }^{3}$ Graduada em Enfermagem. Doutora em Enfermagem e Biociências pela Universidade Federal do Estado do Rio de Janeiro. Enfermeira do Hospital Universitário Pedro Ernesto da UERJ. ${ }^{4}$ Graduada em Enfermagem. Doutora em Enfermagem pela Universidade do Estado do Rio de Janeiro. Docente na Universidade do Estado do Rio de Janeiro. ${ }^{5}$ Graduada em Enfermagem. Mestre em Enfermagem pela Universidade Federal do Estado do Rio de Janeiro. Docente na Universidade do Estado do Rio de Janeiro. ${ }^{6}$ Graduada em Enfermagem. Residente em Terapia Intensiva pelo Hospital Universitário Pedro Ernesto da UERJ

Como citar este artigo:

Souza KA, Paula VG, Bridi AC, et al. Condutas dos Profissionais de Enfermagem Frente os Alarmes dos Ventiladores Mecânicos em uma Unidade de Terapia Intensiva. 2018;8:e2678. [Access___]; Available in:__. DOI: http://dx.doi.org/10.19175/recom.v8i0.2678 


\section{INTRODUÇÃO}

Este estudo tem como tema a fadiga de alarme dos ventiladores mecânicos e as condutas dos profissionais de enfermagem diante deles. A escolha deste tema deve-se à necessidade de uma maior discussão quanto à resposta aos alarmes e ao preparo dos profissionais para atender as demandas do paciente em suporte ventilatório.

A ventilação mecânica (VM) substitui total ou parcialmente a ventilação espontânea. Propicia melhora das trocas gasosas e diminuição do trabalho respiratório, podendo ser utilizada de forma não-invasiva e de forma invasiva, sendo de grande importância na Unidade de Terapia Intensiva ${ }^{(1)}$.

O Instituto Emergency Care Research Institute (ECRI), publica, anualmente, uma lista dos 10 (dez) temas de maior interesse sobre segurança do paciente, sendo alguns deles referentes aos perigos das tecnologias utilizadas na assistência à saúde (TOP 10). Durante alguns anos, a listagem apontou os alarmes como sendo a principal ameaça à segurança do paciente, na área da saúde, superando outras tecnologias da assistência. Nos anos de 2012, 2013 e 2014, os alarmes ocuparam o primeiro lugar e, em 2016, os alarmes foram considerados o segundo lugar da lista. Em 2017, os alarmes perdidos podendo levar dano ao paciente ocuparam o terceiro lugar no ranking ${ }^{(2)}$.

O desenvolvimento das tecnologias de monitorização tem acompanhado o progresso dos cuidados intensivos. Com a finalidade de dar segurança aos doentes graves, os monitores dispõem de alarmes para uma diversidade de variáveis fisiológicas. Assim, a equipe pode ser alertada quanto a mudanças nos parâmetros vitais. Devido à crescente incorporação de tecnologias na unidade de terapia intensiva, o número de alarmes também tem crescido, tornando-se uma questão de preocupação entre pesquisadores e profissionais da terapia intensiva ${ }^{(3)}$.

Os profissionais que trabalham em setores com maior quantitativo de equipamentos eletromédicos e, consequentemente, maior quantidade de alarmes, estão mais suscetíveis à ocorrência de fadiga de alarmes, podendo, na maioria das vezes, mostrar-se indiferente ao soar desses. O fenômeno fadiga de alarme pode ser entendido como o retardo no tempo resposta dos profissionais a eles, podendo os mesmos serem desabilitados, silenciados ou ignorados, a equipe tornando-se insensível aos sons, diminuindo o estado de alerta ${ }^{(4)}$.

O ECRI, em 2006, recebeu cerca de 2200 relatórios relacionados à tecnologia, sendo que, aproximadamente $12 \%$ incluíram a palavra "alarme" no campo de descrição do problema. Desse total, 39\% eram de ventiladores mecânicos. Os relatórios evidenciaram ocorrência de falhas do dispositivo, que colocavam o paciente em risco e que não geram alarme para alertar os profissionais ${ }^{(5)}$.

A Association of Medical Instrumentation AAMI Foundation, em março de 2014, publicou um Webinar com o título "Current Challenges with Ventilator Alarms", que lista uma série de recomendações para melhorar as práticas de segurança dos ventiladores e para lidar com a problemática dos alarmes disparados.

Estudos mostram que os alarmes disparados por VM podem representar de 30 a $33 \%$ de todos os alarmes de uma unidade de cuidados intensivos, sendo os alarmes de elevação da frequência respiratória e de elevação da pressão de vias aéreas os mais prevalentes. Tal fato deve ser motivo de preocupação, uma vez que os alarmes podem estar representando algum problema em relação ao suporte ventilatório empregado ao paciente e que, quando ignorados, predispõem a vida do paciente a uma condição iminente de risco ${ }^{(5-6)}$.

Ao longo do tempo, algumas funções no setor de terapia intensiva referentes à ventilação mecânica deixaram de ser exercida pelo Enfermeiro. Um estudo mostrou que práticas antes específicas da enfermagem, como montagem do ventilador, definição de parâmetros iniciais, aspiração de vias aéreas, acompanhamento gasométrico, desmame ventilatório, entre outros, estão sendo praticadas por profissionais de outras áreas ${ }^{(7)}$. Com os cuidados com o ventilador mecânico sendo exercido por outras categorias profissionais, observa-se um possível distanciamento do enfermeiro, até mesmo no atendimento dos alarmes disparados pelo ventilador. A equipe de enfermagem é a equipe em maior número no ambiente de terapia intensiva, sendo os profissionais que mais participam da vigilância do paciente grave.

Dentre os profissionais que atuam na Unidade de Terapia Intensiva (UTI), o Enfermeiro 
é responsável pelos cuidados diretos ao paciente crítico, conforme Lei no 7498/86, do Conselho Federal de Enfermagem, possuindo inúmeras atribuições em seu dia a dia de trabalho, precisando desenvolver várias atividades, tanto assistenciais quanto gerenciais. É imprescindível que esse profissional esteja capacitado para lidar com os cuidados do paciente em ventilação mecânica. As Diretrizes Brasileiras de Ventilação Mecânica de 2013 descrevem, nos cuidados de enfermagem ao paciente em VM, a checagem dos alarmes e dos parâmetros clínicos ${ }^{(1)}$.

Apesar dos avanços relacionados a investigar o fenômeno fadiga de alarmes, os resultados parecem ainda incipientes no que diz respeito às condutas relacionadas à equipe de enfermagem frente ao soar de alarmes disparados pelos ventiladores mecânicos. Nesse contexto, este estudo teve como objeto de pesquisa, a conduta dos profissionais de enfermagem diante dos alarmes ventilatórios. Foi definido como questão de pesquisa: Quais as condutas dos profissionais de enfermagem diante dos alarmes ventilatórios?

A relevância do estudo está no fato de que os VM utilizados em terapia intensiva estão cada vez mais presentes nessas unidades $\mathrm{e}$ apresentam-se com tecnologias complexas, agregando uma série de sistemas de alarmes, que podem comprometer o estado de alerta da equipe de enfermagem, a qualidade da assistência e a segurança do paciente.

Os objetivos da pesquisa foram: caracterizar quais os alarmes sonoros disparados por ventilador mecânico são mais frequentes na unidade, descrever o tempo estímulo-resposta aos alarmes do ventilador mecânico e analisar as condutas dos profissionais de enfermagem diante dos alarmes ventilatórios.

\section{MÉTODOS}

Trata-se de uma pesquisa do tipo descritiva, com abordagem quantitativa. A pesquisa foi desenvolvida em uma UTI de um hospital universitário da rede pública estadual no município do Rio de Janeiro, possui um quantitativo de 10 leitos, sendo 9 leitos comuns e um leito de isolamento respiratório.

O espaço físico destinado aos 10 leitos e à circulação dos profissionais de saúde e familiares (no horário de visita), ocupa uma área localizada no $5^{\circ}$ andar da Instituição. Há um posto de enfermagem com visão total do VM dos leitos 3,4,5,6,7,8 e vista parcial do VM nos leitos 1,2,9,10.
Todos os leitos da unidade estão equipados com o ventilador mecânico SERVO-S ${ }^{\circledR}$. Ele possui 4 opções de modos ventilatórios: volume controlado (VC); controle de pressão (PC); pressão de suporte (PS) / pressão positiva contínua nas vias aéreas (CPAP) e ventilação mandatória intermitente sincronizada (SIMV). Possui também os seguintes alarmes: frequência respiratória (FR) alta, FR baixa, volume minuto expirado baixo, volume minuto expirado alto, ventilação reserva, bateria em funcionamento, pressão de vias aéreas alta, aumento da concentração de $\mathrm{O}_{2}$, problema na rede de gazes, pressão positiva expiratória final (PEEP) alta, PEEP baixa, entre outros (Manual de instruções do VM).

A unidade conta com uma equipe multiprofissional composta por médicos, nutricionistas, fisioterapeutas, fonoaudiólogos, psicólogos e equipe de enfermagem que contém 13 enfermeiros, 39 técnicos de enfermagem e por ser um hospital universitário com programa de residência, conta com a presença de um quantitativo de 15 residentes em enfermagem em terapia intensiva. A escala de serviço possui seis equipes divididas entre serviço diurno e serviço noturno. No serviço diurno, a equipe é composta por 1 enfermeiro e 5 técnicos de enfermagem e, no serviço noturno, 2 enfermeiros e 5 técnicos de enfermagem.

Como critérios de inclusão foram selecionados todos os profissionais de enfermagem (enfermeiros, técnicos de enfermagem e residentes de enfermagem) que atenderam aos alarmes soados pelos ventiladores mecânicos.

Foram excluídos do estudo, os participantes que compõe a equipe de enfermagem mas não fazem parte da assistência direta a esses pacientes, os profissionais licenciados, afastados e remanejados do quadro de profissionais de enfermagem da UTI.

O Termo de Consentimento Livre e Esclarecido, foi aplicado a todos os profissionais de enfermagem que aceitaram a participação na pesquisa, para autorização da observação durante sua atuação.

Foram obedecidas as normas da Resolução 466/2012, do Conselho Nacional de Ética e Pesquisa, que norteia pesquisas envolvendo seres humanos e respeitados os princípios éticos de autonomia, beneficência, não maleficiência e justiça, submetido ao Cômite de Ética e Pesquisa (CEP) da instituição, aprovação no Certificado de Apresentação para Apreciação Ética (CAEE) 2.046.512. 
A coleta de dados foi realizada através de um instrumento preenchido pela pesquisadora durante a observação não participativa, sendo realizada em momentos distintos, todos durante os plantões diurnos, totalizando 60 horas de observação e registro. A opção de produzir dados somente nos plantões diurnos foi opção da pesquisadora, devido às dificuldades administrativas e operacionais para acessar o cenário de estudo no período noturno.

$O$ instrumento de coleta de dados foi composto pelas variáveis: leito, alarme, profissional, tempo, conduta e observações. Para a definição do tempo estímulo-resposta, foi utilizado um cronômetro e, durante a observação dos leitos, foi selecionado um ponto estratégico em frente aos leitos. Assim, no momento em que um dos alarmes do equipamento eletromédico disparava, era acionado o cronômetro e registrado, no instrumento de coleta de dados, o leito do VM que gerou o sinal do alarme; o profissional que atendeu ao alarme; o tempo estímulo resposta e a conduta do profissional que atendeu $\mathrm{o}$ alarme sonoro.

Para determinação do tempo estímuloresposta, foi considerado o intervalo de tempo registrado entre o soar do alarme até a chegada do profissional à beira do leito do paciente. $O$ tempo estímulo-resposta deixou de ser cronometrado quando atingiu 5 minutos, e foi registrado como alarme sem resposta, fatigado.

Foi definido o limite de 5 minutos, pois, em se tratando de uma parada cardiorrespiratória
(PCR), situação de absoluta emergência, comum entre os pacientes críticos, por exemplo, quanto maior o tempo de resposta ao alarme nesses casos e o retardo no início imediato das manobras de reanimação cardiopulmonar, pior o desfecho neurológico ${ }^{(8-10)}$.

Para o registro da variável do ventilador mecânico responsável pelo disparo do alarme, utilizamos as informações expressas diretamente na interface (tela principal) do ventilador mecânico.

Após a coleta dos dados, os mesmos foram codificados e compilados em um banco de dados informatizado no programa Microsoft Office Excel 2007. Os dados foram tratados com estatística descritiva. Após a observação das condutas dos profissionais, foram descritas e confrontadas com o que há de pré-existente na literatura.

\section{RESULTADOS E DISCUSSÃO}

Foram realizadas 60 horas de observação, sendo em 5 momentos distintos de 12 horas (7h às 19h), no turno diurno. No período de observação, verificou-se uma redução significativa de profissionais por plantão na unidade.

Em 60 horas de observação, foram registrados um total de 128 alarmes disparados, aproximadamente 2 alarmes por hora. Desse total, 67 alarmes do monitor multiparamétrico, 36 de bombas infusoras e 25 de ventiladores mecânicos (Figura 1).

Figura 1- Distribuição dos alarmes disparados na UTI. Rio de Janeiro, RJ.2017.

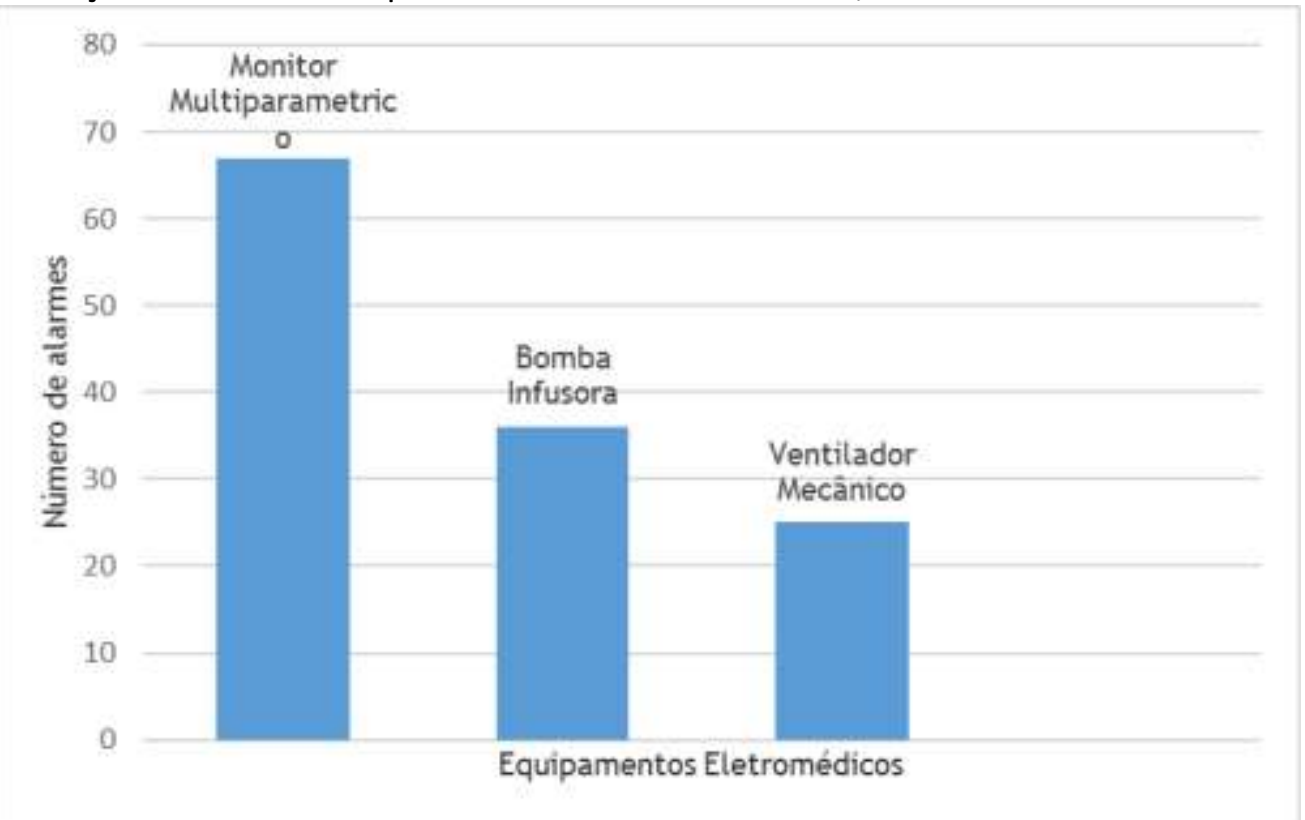


Em relação aos 25 alarmes dos ventiladores mecânicos, 20 foram atendidos e 5 cessaram sem nenhuma intervenção após soarem por 5 segundos. Dos 20 profissionais que atenderam a esses alarmes, $28 \%$ eram enfermeiros, $32 \%$ eram fisioterapeutas, $12 \%$ profissionais da equipe médica e $8 \%$ eram técnicos de enfermagem (Figura 2).

Figura 2 - Categorias profissionais que atenderam aos alarmes do ventilador mecânico. Rio de Janeiro, RJ.2017.

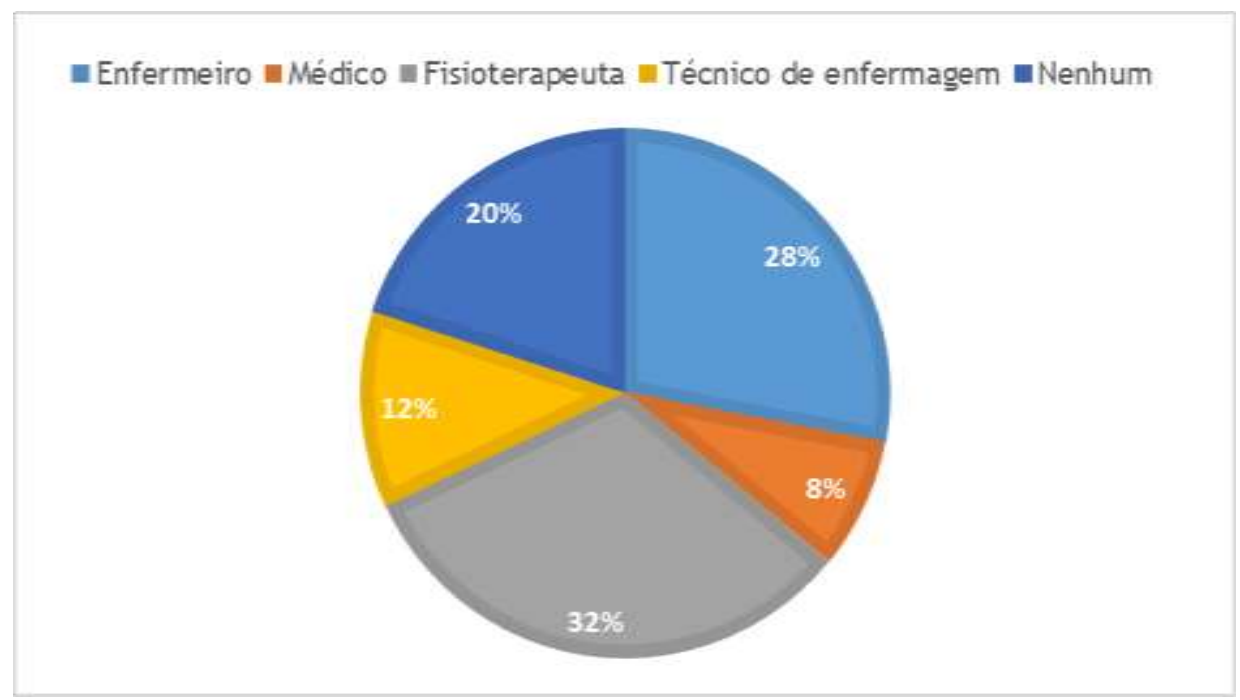

Fonte: Souza (2017).

Dentre os alarmes disparados pelo ventilador mecânico na unidade, os mais prevalentes foram volume minuto expirado baixo
(14), seguido de pressão de vias aéreas alta (7). Também ocorreram os alarmes frequência respiratória alta (2) e apneia (2) (Figura 3).

Figura 3- Tipos de alarmes disparados na unidade pelo ventilador mecânico. Rio de Janeiro, RJ.2017.

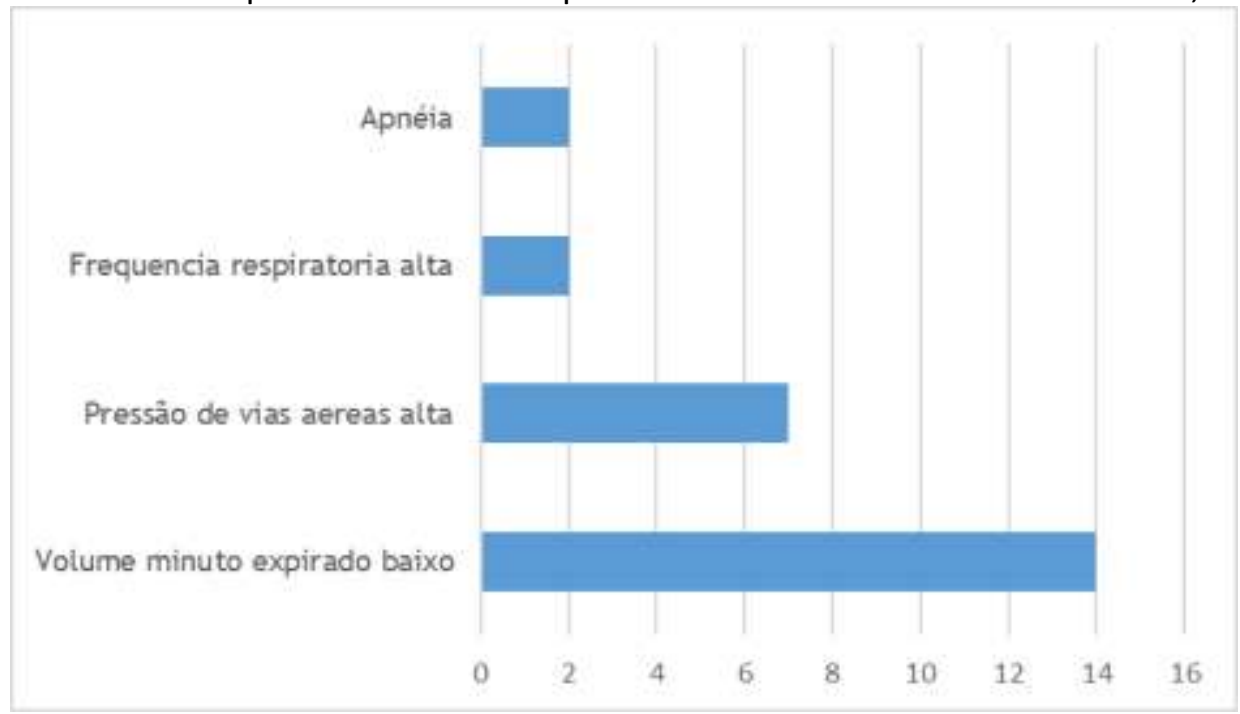

Fonte: Souza (2017).

Neste estudo, foi considerado o tempo estímulo-resposta e as condutas apenas dos profissionais de enfermagem referentes aos alarmes dos ventiladores mecânicos. O tempo estímulo-resposta mínimo para o atendimento desses alarmes foi de 10 segundos. Esse tempo se justifica devido à presença dos profissionais no leito do paciente durante o soar do alarme, seja no momento do exame físico, durante o banho no leito ou acompanhando algum procedimento. O tempo estímulo-resposta máximo para o atendimento do alarme foi de 3 minutos. 0 tempo estímulo-resposta médio foi de 38 segundos e o desvio padrão 52,39 segundos. 
Nenhum alarme soou por mais de 5 minutos, tempo determinado neste estudo para considerar fadiga de alarme.

Apesar do maior número de alarmes ter sido atendido por profissionais de fisioterapia, atendendo a um dos objetivos deste trabalho, foram observadas e registradas as atitudes dos profissionais de enfermagem no atendimento dos alarmes disparados pelos ventiladores mecânicos na UTI. No atendimento de um alarme, foram consideradas todas as condutas realizadas pelo profissional, ou seja, realizou-se mais de uma ação no atendimento de apenas um alarme e todas foram contabilizadas. A conduta mais presente foi a aspiração traqueal (tabela 1).

Tabela 1- Condutas dos profissionais de enfermagem no atendimento aos alarmes dos ventiladores mecânicos. Rio de Janeiro, RJ. 2017.

\begin{tabular}{lcc}
\hline Condutas & Número & Porcentagem \\
& & \\
Aspiração traqueal & 3 & $25 \%$ \\
Mudança de decúbito & 1 & $8,3 \%$ \\
Reposicionamento do circuito & 2 & $16,6 \%$ \\
Ausculta & 2 & $16,6 \%$ \\
Chamar o fisioterapeuta & 2 & $16,6 \%$ \\
Silenciar o alarme & 1 & $8,3 \%$ \\
Ignorar & 1 & $8,3 \%$ \\
\hline
\end{tabular}

Fonte: Souza (2017).

A Resolução da ANVISA (Agência Nacional de Vigilância Sanitária), Resolução da Diretoria Colegiada (RDC) n. 26, de 11 de maio de 2012, descreve que o quantitativo de profissionais de enfermagem na UTI deve ser de, no mínimo, 1 enfermeiro assistencial para cada 10 leitos ou fração em cada turno; e de, no mínimo, 1 técnico de enfermagem para cada 2 leitos em cada turno ${ }^{(11)}$.

Em relação aos profissionais de enfermagem, a unidade não estava de acordo com o preconizado. A coleta de dados foi efetuada durante um período de redução de profissionais por absenteísmo na unidade. Portanto, havia, na unidade, um número menor de profissionais de enfermagem, o que poderia oferecer dificuldades para o atendimento aos alarmes disparados pelos equipamentos eletromédicos. Essa realidade também pode trazer maior desgaste físico e mental dos profissionais em atuação, aumentando os níveis de estresse e colaborando para a fadiga de alarmes na unidade.

Os alarmes dos ventiladores mecânicos mais frequentes na unidade foram volume minuto expirado baixo e pressão de vias aéreas alta. Houve também a ocorrência dos alarmes de frequência respiratória alta e apneia. O alarme volume minuto expirado baixo pode ter como possíveis causas: vazamento ao redor da via aérea artificial; fístula broncopleural; aumento da complacência; vazamento no circuito ou na válvula expiratória; pressão insuficiente nas redes de gazes; defeito do sensor de fluxo; parâmetros inadequados (fluxo ou tempo inspiratório insuficientes, limite de pressão muito baixo ou valor de pressão mínima alta). As possíveis causas para o alarme pressão de vias aéreas alta são: secreções, obstrução ou dobra da via aérea artificial, intubação seletiva, tosse, mordida do tubo orotraqueal (TOT), complacência diminuída, pneumotórax, broncoespasmo, assincronia paciente/ventilador, dobra do circuito, água no circuito, valor limite baixo ${ }^{(12)}$.

Foi identificado que as causas mais frequentes para o acionamento dos alarmes referentes a volume minuto expirado baixo e pressão de vias aéreas alta, foi o acúmulo de secreção e vazamento no circuito. O aumento das secreções pode ocasionar obstrução da via aérea, provocando tosse e, por conseguinte, o aumento da pressão intrapulmonar que, somada à pressão preestabelecida no ventilador, diminui o volume corrente. Tais situações fazem com que o alarme de pressão de via aérea alta e pressão/volume minuto baixo disparem. Alguns estudos corroboram com essa pesquisa, com destaque ao alarme pressão de vias aéreas alta como um dos mais prevalentes ${ }^{(5-6)}$.

O tempo estímulo resposta médio, neste estudo, foi de 38 segundos, sendo o menor tempo 10 segundos e o maior tempo 3 minutos. Mensurar o tempo estímulo-resposta é decisivo para determinar se a fadiga de alarme está presente ou não. Quanto maior for o tempo para intervenção, maior será o risco para o paciente. 
Saber interpretar o que foi sinalizado pelo alarme e entender o perfil de relevância clínica que tal alarme representa para o paciente também merece atenção da equipe. Para muitos profissionais da equipe de saúde, os alarmes são geralmente vistos como inúteis devido à alta incidência de alarmes falsos positivos, ou seja, aqueles sem relevância clínica ${ }^{(4)}$.

O Comitê Europeu de Normalização (CEN) estabeleceu uma classificação dos alarmes em três categorias: prioridade alta, indicando uma situação de urgência (que pode levar imediatamente a um problema vital e exige uma resposta imediata da equipe de saúde); prioridade média, indicando uma situação de perigo (é necessária uma resposta rápida da equipe) e de baixa prioridade, indicando uma situação de alerta (é necessária atenção pela equipe). Em relação ao VM, o estudo do CEN mostra que alarmes de alta prioridade são aqueles relacionados à falha elétrica ou pneumática, ou alta pressão das vias aéreas. Os alarmes de desconexão, apneia, volume minuto expiratório baixo ou concentração alta ou baixa de oxigênio durante a inspiração são consideradas como prioridade média ${ }^{(13)}$.

Observou-se que o alarme pressão de vias aéreas alta, classificado como alta prioridade, apesar de ter sido o segundo mais frequente na unidade, não foi atendido por profissionais de enfermagem, sendo o mais predominante dentre os alarmes que pararam de soar espontaneamente, em até 5 segundos. Esse fenômeno foi citado em outros estudos, que ocorre quando o ventilador mecânico retorna aos parâmetros preestabelecidos sem nenhuma intervenção, sendo essa característica apenas desse equipamento eletromédico ${ }^{(5-6)}$. Portanto, a avaliação do tempo estímulo resposta especificamente para esse alarme foi prejudicada. Para o alarme volume minuto expirado baixo, classificado como média prioridade, todos tiveram atendimento em tempo oportuno, sendo atendidos em até 20 segundos.

A Joint Commission, desde janeiro de 2014, tem apontado os VM como um dos grandes responsáveis por disparar alarmes inconsistentes. Os VM geram um número alto de alarmes sonoros e muitos destes acabam ficando sem resposta dos profissionais. Em parte, isso se deve ao curto período de duração desses alarmes, uma vez que, antes mesmo que possam ser respondidos ou até mesmo percebidos pelos profissionais, silenciam-se automaticamente assim que a variável fisiológica retorne aos valores considerados aceitáveis ${ }^{(5)}$.

Vale ressaltar que, ainda que a variável fisiológica retorne para seus valores aceitáveis e o VM deixe de disparar o alarme, é essencial que a equipe de saúde avalie o que levou ao disparo do alarme e o sincronismo ventilador/paciente com a intenção de prever possíveis problemas que possam interferir na segurança do paciente. Ao parametrizar os alarmes de acordo com os limites de cada paciente, poderá minimizar os alarmes inconsistentes. É fundamental que a equipe de enfermagem seja capaz de gerenciar o uso das tecnologias, de modo a reforçar a qualidade da assistência, com apoio na realização dos cuidados em um ambiente clínico de alta complexidade ${ }^{(14)}$. Neste estudo, nenhum dos 5 alarmes sob esta condição recebeu qualquer atenção dos profissionais enfermagem.

Para o atendimento ao alarme volume minuto expirado baixo, os profissionais de enfermagem tiveram as seguintes condutas: aspiração traqueal, mudança de decúbito, reposicionamento do circuito do VM, ausculta pulmonar e comunicar ao fisioterapeuta as alterações após as condutas que não foram resolutivas.

Como causas de diminuição de volume passíveis de verificação pelos enfermeiros, citamse: desconexões ou fratura dos circuitos; cuff desinsuflado ou furado; piora da complacência e aumento da resistência à passagem do fluxo de ar.

Esses fatores proporcionam o aumento do risco de hipoventilação e hipoxemia para o paciente. De acordo com a literatura, as ações de enfermagem para resolução desse alarme são: verificar o balonete (cuff), a posição e o tamanho do dispositivo ventilatório, auscultar o paciente (verificar a ventilação), checar as conexões e coletores (trocar peças defeituosas), ventilar manualmente o paciente, se necessário, e checar a rede e a necessidade de trocar válvulas redutoras $^{(12)}$.

Com relação ao aumento da pressão de vias aéreas, seria esperado que os enfermeiros identificassem ou verificassem algumas causas: conexão errada das traqueias do circuito com o paciente, obstrução ou dobradura do circuito, intubação seletiva, broncoespasmo, hipersecreção e tampões mucosos (rolhas de secreção); tosse e mordida do tubo orotraqueal(TOT); assincronismo paciente/venti-lador; presença de 
água condensada no circuito e valor limite baixo $^{(12)}$.

As medidas que deveriam ser adotadas são: aspirar secreções, posicionar ou argumentar a troca do tubo orotraqueal (TOT)/ traqueostomia (TQT) (obstrução), aferir a pressão do cuff, tranquilizar o paciente e avaliar o ajuste da sedação, rever parâmetros junto ao médico (volume corrente, frequência respiratória, fluxo, tempo inspiratório/expiratório), ajustar a sensibilidade e os alarmes, corrigir dobraduras do circuito, retirar água condensada do circuito e usar coletores $^{(12)}$.

Em relação às ocorrências de alarmes relacionados, às alterações na frequência respiratória (frequência respiratória alta), acredita-se que os enfermeiros deveriam identificar ou verificar as seguintes causas: comprometimento neurológico do paciente, sedação inadequada, má adaptação ao modo ventilatório, dor ou desconforto, e aumento do trabalho respiratório (exemplo: fadiga muscular em casos de desmame ventilatório), defeito do sensor de fluxo, autociclagem do aparelho, ajuste inadequado da sensibilidade. O paciente com taquipneia persistente pode gerar redução do volume corrente por diminuição do tempo inspiratório com consequente hipoventilação. Nesse caso, a literatura aponta que os enfermeiros podem avaliar a causa, avaliar a necessidade de sedação e/ou analgesia, tranquilizar o paciente, verificar o ajuste da sensibilidade, verificar saturação do paciente, conferir a frequência respiratória do paciente por outro método e comunicar ao médico ${ }^{(12)}$.

Neste estudo, os alarmes frequência respiratória alta e volume minuto expirado baixo tiveram, como conduta dos profissionais de enfermagem, 0 ato silenciar o alarme. Ao desabilitar, silenciar, ignorar ou retardar o temporesposta aos alarmes, os profissionais de saúde retardam também o tempo para a implementação de condutas terapêuticas ou alterações significativas nos pacientes, que podem não ser percebidas, não oferecendo segurança no seu acompanhamento. A utilização incorreta dos equipamentos, alarmes inoperantes, com parâmetros inadequados e baixo volume estão relacionados a eventos adversos com equipamentos eletromédicos ${ }^{(15)}$. Na avaliação da segurança dos VM, deve-se considerar as características dos alarmes e mecanismos de segurança.
O alarme de apneia indica interrupção ou redução da ventilação espontânea e podem ter como possíveis causas: comprometimento neurológico do paciente, sedação inadequada, ajuste inadequado da frequência respiratória, da sensibilidade ou do tempo de apneia. Diante dos alarmes de apneia, deve-se avaliar o paciente, ventilar de modo controlado, verificar se o VM detecta as ventilações espontâneas do paciente, rever sedação ${ }^{(12)}$. Os profissionais da unidade que atenderam este alarme comunicaram ao fisioterapeuta e, juntos, reavaliaram o modo ventilatório.

\section{CONCLUSÃO}

Os achados apontaram que os alarmes mais frequentes: volume minuto expirado baixo e pressão de vias aéreas alta, as condutas realizadas pelos profissionais de enfermagem, de acordo com a literatura, não foram suficientes para a resolutividade dos problemas apresentados. 0 tempo estímulo-resposta dos profissionais teve como tempo médio, 38 segundos, apontando que os alarmes atendidos foram em tempo oportuno.

A redução da equipe de enfermagem na escala de serviço, no período da coleta de dados, foi uma limitação do estudo, assim como um número menor de pacientes em ventilação mecânica na unidade. Entende-se que, com o número maior de pacientes nessa condição e uma equipe com número adequado, os registros de alarmes e as condutas apresentariam números e características diferentes. Outra limitação do estudo foi o fato de ter apenas uma pesquisadora cronometrando o tempo, ficando impossibilitada de proceder à observação, caso outro alarme soasse ao mesmo tempo. Pretende-se, com esses resultados, contribuir para implementar treinamento aos profissionais de enfermagem, de modo que possam atender, em tempo hábil e de maneira segura, os alarmes dos ventiladores mecânicos, bem como a adequada tomada de decisão quando ao soar desses alarmes, o gerenciamento e parametrização destes.

\section{REFERENCES}

1- Barbas CSV, Ísola AM, Farias AMC. Diretrizes brasileiras de ventilação mecânica. São Paulo: AMIB; 2013.

2- ECRI Institute. Top 10 Health Technology Hazards for 2017 [Internet]. 2017[cited in 2017 Out 8]. Disponível em: https://www.ecri.org/ Resources/Whitepapers and reports/Haz17.pdf 
3- Bridi $A C$, Silva $R C L$, Farias $C C P$, Franco $A S$, Santos VLQ. Tempo estímulo-resposta da equipe de saúde aos alarmes de monitorização na terapia intensiva: Implicações para a segurança do paciente grave. Rev Bras Ter intensiva 2014 jan/mar;26(1):28-35. DOI: 10.5935/0103-507X. 20140005

4- Graham KC, Cvach M. Monitor alarm fatigue: Standardizing use of physiological monitors and decreasing nuisance alarms. Am J Crit Care. 2010 Jan;19(1):28-37. DOI: 10.4037/ajcc2010651

5- Passamani RF, Santos F, Schutz V, Silva CRL, Louro TQ, Silva RCL. Usabilidade de alarmes clínicos de ventiladores mecânicos em cuidados intensivos. Ciênc Cuid Saúde. 2016 abr/jun;15(2):220-6. DOI: 10.4025/cienccuid saude.v15i2.29234

6- Santos F, Silva RCL, Ferrão PPSA, Ribeiro AS, Passamani RF. Fadiga de alarmes de equipamentos eletromédicos em terapia intensiva. Rev Enferm. 2014 mar;8 (3):687-94. DOI: $\quad 10.5205 /$ reuol.5149-42141-1-SM.0803201 $\underline{425}$

7- Barreiro Filho RD, Santiago LC, Silva RFA, Cunha KCS. $O$ paciente submetido à ventilação mecânica e o cuidado de enfermagem que emerge da prática assistencial. In: Anais do 61을 Congresso Brasileiro de Enfermagem, 2009; Fortaleza. Fortaleza: ABEn; 2009. p. 5012-26.

8- Gonzalez MM, Timerman S, Oliveira RG, Polastri TF, Dallan LAP, Araújo $S$, et al. I Diretriz de ressuscitação cardiopulmonar e cuidados cardiovasculares de emergência da sociedade brasileira de cardiologia: Resumo executivo. Arq Bras Cardiol. 2013 fev;100(2):105-13. DOI: 10.5935 /abc.20130022

9- Tallo FS, Moraes Junior R, Guimarães HP, Lopes RD, Lopes AC. Atualização em reanimação cardiopulmonar: Uma revisão para o clínico. Rev Soc Bras Clín Méd. [Internet]. 2012 [acesso em 2017 maio 5]; 10(3):194-200. Disponível em: http://files.bvs.br/upload/S/1679-1010/2012/ v10n3/a2891.pdf

10- Vanheusden LMS, Santoro DC, Szpilman D, Batista CO, Correia LFB, Cruz Filho FES. Conceito fase-dependente na ressuscitação cardiopulmonar. Rev SOCERJ [Internet]. 2007 [acesso em 2017 mar 15];20(1):60-4. Disponível em: http://sociedades.cardiol.br/soceri/revista/ 2007 01/a2007 v20 n01 art09.pdf

11- Ministério da Saúde. Agência Nacional de Vigilância Sanitária. Resolução no 26, de 11 de maio de 2012. Dispõe sobre a alteração da RDC 07de 24 de fevereiro de 2010. Diário Oficial da União. 2010 maio.

12- Cintra EA, Nishide VM, Nunes WA. Assistência de enfermagem ao paciente gravemente enfermo. 2. ed. São Paulo: Atheneu; 2013.

13- European Committee for Standardization. Lung ventilators - Part 1: Particular requirements for critical care ventilators - NENEN 794-1:1997. British: British; 1997.

14- Gomes ATL, Assis YMS, Ferreira LL, Bezerril MS, Chiavone FBT, Santos VEP. Tecnologias aplicadas à segurança do paciente: Uma revisão bibliométrica. Rev Enferm CentOeste Min. 2017 jul/dez;7:e1473. DOI: 10.19175/ recom.v7i0.1473

15- Korniewicz DM, Clark T, David Y. A national online survey on the effectiveness of clinical alarms. Am J Crit Care [Internet]. 2008 [acesso em 2017 nov 10];17(1):36-41. Disponível em: https://www.ncbi.nlm.nih.gov/pubmed/1815 $\underline{8387}$

Note: Research report presented at the conclusion of the Specialization Course in Nursing Residency Modality, of the Residency Program in Intensive Care Nursing.

Recebido em: 16/12/2017

Aprovado em: 04/06/2018

Endereço de correspondência:

Kamila Azevedo de Souza

Rua Visconde de Santa Isabel, 143 - Apt 501

CEP: 20560120 - Rio de Janeiro/RJ - Brasil

E- mail: kamilaazevedoenfa@gmail.com 